\title{
WEIRD Testbeds with Fixed and Mobile WiMAX Technology for User Applications, Telemedicine and Monitoring of Impervious Areas
}

\author{
Silvano Mignanti \\ Sapienza University of Rome \\ Italy \\ silvano.mignanti@dis.uniroma1.it \\ Mario Castellano \\ INGV - Osservatorio Vesuviano \\ Italy \\ castellano@ov.ingv.it \\ Mariarita Spada \\ Wind \\ Italy \\ mariarita.spada@mail.wind.it \\ Paulo Simoes \\ University of Coimbra \\ Portugal \\ spsimoes@dei.uc.pt
}

\author{
Gabriele Tamea \\ Sapienza University of Rome \\ Italy \\ gabriele.tamea@dis.uniroma1.it \\ Antonio Cimmino \\ Alcatel Lucent \\ Italy \\ antonio.cimmino@alcatel-lucent.it \\ Pedro Miguel Neves \\ Portugal Telecom Inovacao \\ Portugal \\ est-p-neves@ptinovacao.it
}

\author{
Ilaria Marchetti \\ Sapienza University of Rome \\ Italy \\ ilaria.marchetti@dis.uniroma1.it \\ Fausto Andreotti \\ Italtel \\ Italy \\ fausto.andreotti@italtel.it \\ Giada Landi \\ Centro Pisa Ricerche \\ Italy \\ g.landi@cpr.it \\ Kostas Pentikousis \\ VTT \\ Finland \\ kostas.pentikousis@vtt.fi
}

\begin{abstract}
Wireless Metropolitan Area Networks based on IEEE 802.16d/e standards are soon to be deployed in several countries. However, there is lack of published literature with results from actual testbeds. This paper introduces the work done in the EU Sixth Framework Programme Project WEIRD to design and set up WiMAX testbeds in four EU countries. We describe the methodlogy followed, detail our implementation and present results from the testbeds, as deployed in the first phase of WEIRD. The testbeds are used to demonstrate how WiMAX technology can be used to extend the connectivity of the panEuropean data communications network (GEANT2) to isolated and impervious areas and, furthermore, to assure end-to-end quality of service to novel applications.
\end{abstract}

\footnotetext{
Permission to make digital or hard copies of all or part of this work for personal or classroom use is granted without fee provided that copies are not made or distributed for profit or commercial advantage and that copies bear this notice and the full citation on the first page. To copy otherwise, to republish, to post on servers or to redistribute to lists, requires prior specific permission and/or a fee.

TRIDENTCOM 2008, 17th- 20th Mar 2008, Innsbruck, Austria. Copyright (C) 2011- 2012 ICST ISBN 978-963-9799-24-0 DOI 10.4108/tridentcom.2008.3157
}

\section{Categories and Subject Descriptors}

C.2.1 [Computer-Communication Networks]: Network Architecture and Design - wireless communication

\section{General Terms}

Design, Experimentation, Verification.

\section{Keywords}

WiMAX, QoS, Research Networks, GEANT2, testbed

\section{INTRODUCTION}

The main objective of the WEIRD [19] project is to develop research testbeds using the WiMAX technology in order to allow the connection of isolated and impervious areas to the pan-European research and academic network, GÉANT2 [3]. GÉANT2 is the seventh generation of such a kind of pan-European networks.

The technology used in such project is the WiMAX one. WiMAX, shorthand for "Worldwide Interoperability for Microwave Access", is a telecommunication technology mainly aimed for long range point-to-point and point-to-multipoint transmissions that uses the range of frequencies between 2 and $11 \mathrm{Ghz}$. It is described in the IEEE standards 802.16d [4] and 802.16e [5]: the former defines the physical (PHY) and Medium Access Control 
(MAC) layers for fixed networks, while the latter describes the extensions for mobility support.

Due to the wide range of WiMAX features, an appropriate definition of the possible scenarios is crucial in order to analyze the performance of the WEIRD architectural and algorithmic solutions. The scenarios considered draw their requirements from the final users participating in the project, such as volcano monitoring observatories, telemedicine centres, and forest fire prevention centres. Clearly, these users introduce diverse requirements to the WEIRD architecture. They have different environmental characteristics, traffic load demands and typical conditions, and call for different service configurations and QoS solutions. In addition, the WEIRD project considers both fixed and mobile WiMAX, in the first and second phase, respectively.

Central to the success of the reference architecture is the deployment of testbeds in four European countries (Finland, Italy, Portugal and Romania), and their interconnection via GEANT2. The aim is to validate the architecture and demonstrate its viability via measurable results. Each testbed is devoted to study and analyze WEIRD solutions for a specific scenario using the related technologies and its associated applications.

The paper is organized as follows. Section II motivates and overviews the WEIRD network architecture including the signalling among different architectural entities. Section III details the prospective applications that the WEIRD architecture has to support. Then, Section IV describes relevant scenarios, and Section V presents the four testbeds and our results. Section VI concludes this paper outlining the main next steps in the remaining duration of the project.

\section{WEIRD REFERENCE ARCHITECTURE}

In recent years, network architectures have become more complex due to services differentiation, the increasing number of users that must be supported, and the introduction of heterogeneous technologies. Major standardization bodies, such as ETSI/TISPAN, ITU-T, and 3GPP, have tried to define an architecture that can cope with this rising level of network complexity in an effective manner.

The WiMAX Forum through its Network Working Group has standardized an architecture with several entities and reference points, called Network Reference Model (NRM). The WiMAX Forum NRM comprises two main parts: the Access Service Network (ASN) and the Connectivity Service Network (CSN). Radio access is provided by the ASN components, i.e. Base Stations (BS) and one or more ASN Gateway (ASN-GW), while IP connectivity is provided by CSN.

\subsection{Architecture overview}

The WEIRD architecture has been designed based on a multiplane structure. Vertically, it is partitioned in two strata: (a) the Applications and Services Stratum, which contains the functionalities related with management and control of applications, independently of the network transport layer, and (b) the Transport Stratum, which manages and controls available resources. The

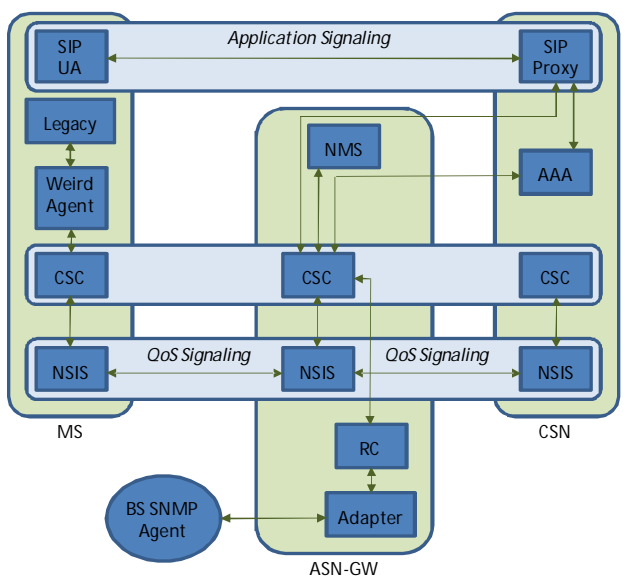

Figure 1. WEIRD Architecture - Control Plane

latter is also responsible for data flow through the network infrastructures.

Horizontally, the WEIRD architecture is composed of the Management Plane, which is responsible for medium- and long-term tasks, such as QoS provisioning through the Network Management System (NMS), and traffic management; the Control Plane, which provides the functionalities for short-term operations, including QoS reservations; and the Data Plane, which is responsible for the transfer of user/application data.

The WEIRD Control Plane is illustrated in Fig. 1

Although it is based on the WiMAX Forum NRM, introduced above, the WEIRD Control Plane includes several new modules to support real time services with QoS differentiation efficiently. With respect to supported applications, both SIP and non-SIP applications are envisaged. We shall refer to non-SIP applications as "legacy applications" in the remainder of this paper. For SIP-based applications, the SIP User Agent (SIP UA) in the Mobile Station (MS) communicates directly with the SIP Proxy in the CSN. Regarding legacy applications, the WEIRD Agent module has been defined for the MS to adapt and configure the required QoS parameters for them.

The Connectivity Service Controller (CSC) modules, located in all the entities of the NRM, have the most important functionalities of the system in terms of QoS control. Since WEIRD is focused on the ASN segment, the CSC at the ASN (CSC_ASN) is the main coordination point for QoS functionalities, such as resources allocation and admission control in both the ASN and the WiMAX segments.

The SIP Proxy extracts the QoS parameters from the SIP/SDP messages, performs user authentication and authorization with the AAA module, and forwards the collected QoS information towards the CSC_ASN using a Diameter $\left(\mathrm{Gq} / \mathrm{Gq}^{\prime}\right)$ [15] interface. The CSC at the MS (CSC_MS) communicates with the WEIRD Agent to obtain the QoS parameters required by legacy applications. CSC_MS provides this information to the main QoS coordination point, the CSC_ASN. On the other hand, the CSC at the CSN (CSC_CSN) receives the QoS reservations requests from the CSC_ASN and establishes the QoS paths in the core 
network. Additionally, CSC_ASN has an interface with the NMS for medium- and long-term management functions, such as QoS provisioning, as mentioned above. Communication between different CSCs is done using NSIS, detailed in the next subsection.

CSC_ASN is also responsible to trigger the Resource Controller (RC) module on the ASN-GW. Since all functionalities related with the WiMAX link are managed and controlled by RC, it can be seen as the WiMAX link manager. RC acts as a generic layer between the upper modules of the architecture and the lower ones. RC plays an important role as it abstracts all WiMAX technology related functionalities for the upper-layer modules, thus guaranteeing hardware independence for the entire architecture.

One of the most important tasks of RC is to manage QoS on the WiMAX link, i.e. Service Flow (SF) Management. RC needs to handle the creation, modification and deletion of SFs in the WiMAX link, as well as the associated Service Classes (SCs) and Convergence Sublayer (CS) Classifiers (CSCl). With respect to the $\mathrm{CSCl}, \mathrm{RC}$ currently supports Ethernet, IPv4 and IPv6 convergence sublayers. To manage the SFs and their status, both a static and a dynamic model have been defined and implemented. The static model is used for medium- and long-term QoS reservations, triggered by the NMS. In this case, each SF must transition between different states, starting with "Provisioning", moving to "Admitted", and so on until it achieves "Active" status. Under the dynamic model, a SF can be directly activated without traversing all states. This model is suitable for real-time services that require instantaneous allocation of SFs on the WiMAX air link.

To enforce the QoS decisions on the WiMAX system, the RC triggers the Adapter module on the ASN-GW. Currently, the Adapter interfaces with the WiMAX BS using SNMP [11], [18]. Therefore, it has to translate the received QoS reservation requests from the RC to the specific objects of the WiMAX BS Management Information Base (MIB) and then issue an SNMP command to the latter. Among all modules defined on the WEIRD architecture, the Adapter is the only one that is dependent on the specific WiMAX equipment in use.

\subsection{Signaling Protocols}

The WEIRD system provides support for a wide range of applications with different QoS requirements, offering mechanisms to coordinate application signalling with the WiMAX resource allocation and the NSIS signalling in the wired core network in order to guarantee a coherent end-to-end QoS signalling along the full path.

The flexibility of NSIS, through the support of different signalling schemes, i.e. end-to-end, edge-to-end, and edge-toedge, allows applications with different signalling capabilities to interact with the WEIRD framework. Following an IMS-based approach, SIP applications are supported through a direct interaction between the SIP Proxy and the CSC on the ASN-GW. In this case, the application description is retrieved by the SIP Proxy from the SDP messages during session establishment and capabilities negotiation, and the ASN-GW acts as the initiator of the NSIS signalling towards the core network.
Legacy applications without standard signalling capabilities can use the WEIRD Agent in order to describe their QoS requirements and request resource allocations. NSIS signalling is initiated directly at the client side and it is coordinated by the CSC on the MS. It should be noted that both approaches follow a network-initiated mechanism for WiMAX resource reservation, as required by the IEEE 802.16 specifications, since the actual WiMAX SF management is entirely handled by the ASN-GW.

NSIS through NSLP is able to support heterogeneous networks by the definition of a set of QoS models that describe the related QoS-enabled domain taking into account the underlying network technology. Each QoS model specifies both the Resource Management Function (RMF) to provide the desired QoS, and the QoS parameters included in an object called QSPEC (QoS Specification) [2].

A specific WiMAX QoS model has been defined. The related QSPEC contains all the parameters for SF configuration. Suitable mechanisms for QoS parameters mapping have been specified in order to allow the interoperability with external domains characterized by different QoS metrics.

The RMF includes signalling scenarios for both SIP and legacy applications. In the first case the ASN-GW is the QoS NSLP Initiator (QNI) and handles the NSIS messages with the QoS parameters received through the application layer signalling and the SIP Proxy. In the second case the MS has the role of the QNI and the application QoS requirements are retrieved directly on the client side.

\section{APPLICATIONS}

WiMAX aims at supporting large number of scenarios, providing fast wireless broadband access for a wide range of applications with QoS guarantees over the last hop. In the extended architecture proposed by the WEIRD project, applications can be categorized into two classes. The first class includes applications that can be adapted to use WEIRD-defined services: we call these applications "WEIRD-aware", and typical examples are the SIPbased modules. The second class includes legacy applications, which cannot be updated but can use WEIRD services through an Agent.

Another classification scheme partitions application based on whether they use SIP signalling for session management or not. For session-based (SIP) applications, the WEIRD architecture aims at supporting two different QoS models: QoS assured, i.e. a session/flow can be established only if the requested/required QoS can be guaranteed; and QoS enabled model, the availability of QoS resources does not affect the success of a session setup, but it may affect the effective level of QoS associated with it. In both cases, the reservation process is driven by a SIP Proxy, leaving the SIP endpoints to participate in the precondition verification mechanism (if used).

Dedicated WEIRD software modules running on the client side (MS) have been developed to support applications that are not SIP-based: Table 1 summarizes the applications types that the WEIRD system can support. 
Table 1. WEIRD Application Categories

\begin{tabular}{|l|l|l|}
\hline $\begin{array}{c}\text { SIP- } \\
\text { based }\end{array}$ & \multicolumn{1}{|c|}{ WEIRD-aware } & Legacy \\
\hline Yes & $\begin{array}{l}\text { Customizable SIP application, } \\
\text { using some SIP/SDP extension(s) } \\
\text { in order to support a specific QoS } \\
\text { model. }\end{array}$ & $\begin{array}{l}\text { COTS SIP clients. QoS } \\
\text { enabled model can be } \\
\text { provided (at least) with BE } \\
\text { quality. }\end{array}$ \\
\hline No & $\begin{array}{l}\text { Customizable application } \\
\text { including a WEIRD API. }\end{array}$ & $\begin{array}{l}\text { Agent is needed to use } \\
\text { WEIRD services. }\end{array}$ \\
\hline
\end{tabular}

\subsection{Environmental Monitoring Applications}

We are interested in both fire and volcano monitoring, which make use of data and video streaming from sensor networks and cameras for the respective user communities. As far as concerns fire prevention application is designed to support both detection and prevention. Additionally, the application is expected to increase security against arsonists. As far as concerns volcano monitoring the integration of permanent and temporary seismic networks gives more complete information about the seismic patterns occurring before and during a crisis. WiMAX could be very useful for downloading data acquired from remote stations of the mobile seismic network to the acquisition centre, thus minimizing the time lag between the acquisition of data and their availability to the scientific community.

\subsection{Telemedicine Applications}

Telemedicine applications include high-resolution video and data streaming from medical equipment, for the clinical user communities. This is one of the areas where WiMAX may offer at reasonable costs the necessary bandwidth in mobility conditions for applications like: Tele-diagnosis (remote diagnosis), Emergencies (remote diagnosis in an emergency situation, i.e. real time response needed), Remote second opinion (a specialist opinion is needed but the specialist is available only in remote area) and Patient follow-up (patients followed by a specialist visit in an authorized remote medical centre where results and images are collected and forwarded to the principal centre).

\subsection{Generic User Applications}

General-purpose applications are also supported and can be employed in more than one of the scenarios discussed in the following section. They include voice over IP (VoIP), videoconference over IP, and video-streaming for generic usage in monitoring and content diffusion. All these applications require the availability of a broadband connection. When we consider mobility and scenarios involving remote and impervious areas, one can acknowledge that such applications can hardly be used. For this reason, voice and video over IP applications will also be used to test WEIRD solutions and to demonstrate the benefits offered by the WiMAX.

Table 2 summarizes the application types, the level of QoS required [9], expressed in terms of the usual set of parameters (i.e. bandwidth, max delay, jitter and loss), and the mapping with the WiMAX class of service $(\mathrm{CoS})$. It is shown that these five
Table 2. WEIRD applications and QoS requirements

\begin{tabular}{|c|c|c|c|c|c|}
\hline 莺 & $\sum_{i=1}^{\infty}$ & 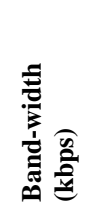 & 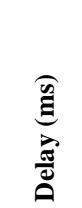 & 氖 & 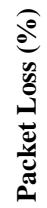 \\
\hline $\begin{array}{l}\text { VoIP } \\
\text { Videoconference }\end{array}$ & $\begin{array}{l}\text { UGS or } \\
\text { ertPS }\end{array}$ & $64-384$ & 150 & 30 & $1-3$ \\
\hline $\begin{array}{l}\text { RT Streaming of } \\
\text { Video / Data }\end{array}$ & rtPS & $5-2000$ & 5000 & na & 5 \\
\hline FTP Data Transfer & nrtPS & $\begin{array}{c}10- \\
2000\end{array}$ & 15000 & na & 0 \\
\hline $\begin{array}{l}\text { HTTP Data } \\
\text { Transfer }\end{array}$ & $\mathrm{BE}$ & $>500$ & $\begin{array}{l}2000 \\
\text { /page }\end{array}$ & na & 0 \\
\hline
\end{tabular}

classes of service (UGS, rtPS, ertPS, nrtPS and BE) could easily fit the requirements of each kind of WEIRD application.

\section{TESTBED SCENARIOS}

\subsection{Environmental Monitoring}

\subsubsection{Volcano Monitoring}

Active volcanoes represent one of the highest natural risks in the world. Volcano eruptions can cause heavy damages to houses and facilities, as well as the death of the inhabitants living near the volcanoes. For these reasons, volcano monitoring centers deem essential to have continuous information about volcanic activity for a maximum possibly affidable eruption forecasting [16].

A modern volcano monitoring system is based on geophysical and geochemical sensor networks. Among all the methods, seismology is probably the most useful one of the entire surveillance system owing to the occurrence, before an eruption, of a large variety of seismic signals.

The Osservatorio Vesuviano (INGV-OV, Italy), has the task to make the continuous monitoring of the Neapolitan active volcanoes (Vesuvius, Campi Flegrei and Ischia Island). These volcanoes rise in a densely populated area (Naples and its surroundings) representing a very high risk. At present, Campi Flegrei caldera shows the major dynamics. In fact, the area is characterized by slow crust subsidence or uplift (bradyseism) accompanied by shallow seismicity. In the last fifty years, four crises occur: 1969-1972, 1982-1984, 2000, and 2004-2006 [10]. The INGV-OV seismic monitoring system deployed on Vesuvius and Campi Flegrei areas is based on a Permanent Seismic Network (Fig. 2) which data are in real-time transmitted to the Acquisition Centre in Naples by means of different type of communications (e.g. UHF FM radio link, UHF RS-232 radiomodem link, dedicated phone line) [1]. Although data collected with Permanent Seismic Network only may be insufficient to study in detail the seismic behaviour during a crisis. In these events, a Temporary Seismic Network is quickly deployed on the volcanic area to improve the permanent one. This is the episode of Vesuvius, after the M=3.6 earthquake occurred on 1999 (the 


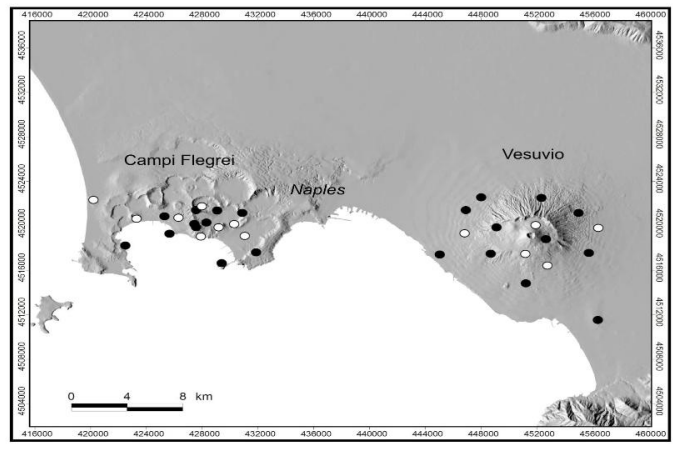

Figure 2. Map of the areas where black and white dots show the Permanent and Temporary Networks, respectively.

stronger earthquake in the last fifty years; some temporary stations are yet installed) and Campi Flegrei, with the recent 2004-2006 crisis (Fig. 2). The temporary network is composed by portable digital seismic stations with local recording on removable high-capacity media. The local recording is a good solution that allows deploying the temporary stations in the best places to both integrate and optimize the permanent network. Nevertheless, the local recording needs a periodic check to retrieve the data and does not allow a real-time integration with the permanent network data.

A solution can be obtained using the LAN output interface of the temporary stations connected to a WiMAX communication link. In this way, the data gathered by the portable devices can be downloaded directly from the Acquisition Centre reducing the time delay between the data recording and their availability. The possibility to integrate near-real-time the seismic data of both permanent and temporary networks during a volcanic crisis is a very important step in helping to define a reliable scenario for a correct eruption forecasting. Moreover, WiMAX connectivity can be useful to other critical applications during a volcanic emergency, such as voice communications between the Acquisition Centre and the teams involved in field activities. In particular, in case of crisis, it will become crucial an intelligent management of the available bandwidth supplied by the WiMAX channel: for example, it could be necessary to assign more bandwidth or higher priority to VoIP message exchange between operators but, at the same time, guarantee some bandwidth to the sensors and cameras. In few words, resource control and QoS guarantees are required in crisis scenarios. The WEIRD architecture is the addon to the WiMAX technology making it possible.

\subsubsection{Fire Prevention}

For decades, forest fires have been a major disaster for rural populations in extensive areas of southern Europe, such as Portugal, Spain, southern France, Greece and Italy. In addition to the most immediate and tragic consequences - loss of lives, loss of properties - there are also enduring problems, such as depletion of resources, ecological impoverishment and deterioration of quality of life.

In spite of large investments, both by the national governments and the local administrations, fire prevention and fire fight efforts have been largely unsuccessful. The difficulty to guarantee early detection of the fire has been one of the main causes of this situation. This is especially true in areas which are scarcely populated or difficult to reach. In these areas, fire detection is usually made by human spotters - atop surveillance towers and planes - searching for smoke and communicating their findings to centralized control centres. Clearly both methods are not particularly cost-efficient:

Communication between surveillance towers and control centres is one of the problems within this scenario. Technologies such as ADSL or 3G/UMTS are not available in remote forests, and solutions such as GPRS, GSM or UHF radio-links can not provide the bandwidth required for more automated fire detection, assuming we are willing to ignore for a moment coverage limitations.

WiMAX can help to change this scenario. Due to its characteristics in terms of range, bandwidth and adaptability to environmental conditions, WiMAX is an interesting choice to provide connectivity to remote sensors and monitoring systems, capable of effectively providing early detection of fire in a more efficient and cost-effective manner. Cameras mounted in surveillance towers can automatically scan a 360 degrees area, with $20-40 \mathrm{~km}$ radius, and send collected data (live video streaming, infrared sensors, automated pre-detection of potential fires, location data, meteorological data) to control centres, where prospective fires are further investigated (for instance remotely pointing and zooming the cameras to the suspect area). This solution is costeffective (the number of required human operators is drastically reduced) and efficient (data received by the control centre is more precise and can lead to instant location of the fire, by triangulation and association with geographical information systems).

During fire fighting, WiMAX can also support better communication between involved partners, for instance allowing the instant exchange of data between fire-fighters and the control centre (GPS-location of the fire-fighters, meteorological data, images of the fire, instructions from the control centre, etc.). As in the volcano crisis case, also in this fire prevention one, in real time crisis scenarios, an intelligent managing of channel resources, i.e. resource management for QoS guarantees is mandatory and this is the role covered by the WEIRD architecture.

\subsubsection{Health}

E-health is one of the areas where WiMAX technology enhanced with the potentialities provided by WEIRD can substantially contribute to improve the daily activities and thus contributing to enhance the quality of life in Europe.

Hereafter some examples are provided of advanced medical services that broadband wireless data and image transmission would make it possible:

- Remote follow-up: today patients travel to far-away hospitals to be followed-up after therapies or chirurgical interventions.

- Remote diagnosis: need to transmit urgent data in order to make an immediate basic diagnosis. Two sub cases 
are envisaged: Data is collected in a fixed place or data is collected on a moving medium, e.g. an ambulance.

- Need to intervene on non transportable patients may require off-air transmission of critical data or images (e.g. last ultrasound scan, PET, real-time video).

- Remote monitoring: today elder people are remotely monitored when at home, not when outside.

- Remote assistance: patients dismissed by hospitals often need to be reminded about therapies and medicines wherever they are.

The most interesting application of WiMAX is to allow connectivity to doctors in the field where usually there is lack of wired network access (e.g. the patient premises or during the way to the hospital).

The WEIRD e-health application is composed by three systems (medical devices, PC unit and Customer Premise Equipment CPE) working together with the aim of sending images, video and data from/to a client of a doctor on the field in case he needs remote supports by a remote centre. The remote medical specialist should use directly the server or be connected to the server by a wired or wireless network. The most interesting application of WiMAX is to allow access to the field doctors that have not availability of wired internet access on the remote location, or during the path to the hospital.

E-health can use voice and video over IP applications in order to support real-time communication in case of emergency. In addition, the same applications could enhance services such as remote assistance and patient's monitoring as well as data distribution and collection. Scalable Collaborative platforms, having web interfaces, could be deployed by the medical authorities and as well as easy administrated.

As a result of the above introduced scenarios, e-health applications represent excellent drivers for both broadband and mobility requirements. The need to have an always-on broadband link from the ambulance to the serving hospital is a strong requirement for both WiMAX and 3G-HDSPA/LTE architectures. The availability of this kind of channel is an issue that cannot be solved with traditional 3G/GPRS deployment.

\subsubsection{Generic User Applications}

Scenarios for generic user applications are heterogeneous and can include different user mobility conditions (slow or fast mobility) and different applications and requirements. In our approach we focused on VoIP and FTP transfer. In fact VoIP is an emerging application growing more and more in interest for both users and network operators while FTP is a consolidated application used since the early days of network usage.

\section{TESTBED IMPLEMENTATIONS AND RESULTS}

The scientific users' community of the project includes organizations involved in fire prevention/protection and health/medical associations.

The technical solutions developed within the WEIRD project have been implemented, tested and validated in four test-beds deployed in the following European nations: Finland, Italy, Portugal and Romania; the interconnection between test-beds is be based on the pan-European GEANT2 research network. The test-beds have different profiles and technology according to the aforementioned scenarios. Moreover, every test-bed is specialized in order to highlight certain technical solutions. Thus, the WEIRD Project has moved in a considerably large and rich domain for validating the developed solutions. This is considered as one of the most valuable and unique assets of the project.

Even though not all of the application scenarios and technical enhancements are developed in every test-bed, the project has developed solutions for each test-bed to demonstrate the interworking of the selected applications in larger scale e.g. VoIP transmission through the pan-European research network; here extensive information on test-beds and their best vocation in the context of the WEIRD project are provided.

The WEIRD project uses different equipment based on the WiMAX technology rel. $\mathrm{d}$ and rel. e, in order to realize very complex architectural schemas, including both fixed and mobile scenarios. In fact, WiMAX fits naturally into the strategy for user centric broadband that is fully focusing on the standard IEEE802.16e, which is considered as the most versatile, high performance version of the standard capable of supporting a comprehensive range of both fixed and nomadic or low mobility applications. In the WEIRD project also the WiMAX IEEE802.16d will be used in order to realize some components of the different test beds: in particular many important aspects concerning the WiMAX d capabilities have been anticipated using different equipment already available on the market; as concern the possibility to test also some applications based on the WiMAX e capability, above all the mobility aspects, in the different test it will be evaluated the opportunity to introduce the new solution based on the Alcatel WiMAX 9100 product in order to realize more complex experimental schemas: this decision has been taken according to some different issues first of all the equipment and the frequencies availability for each involved testbed and the time frame evaluation to perform all the foreseen experimental activities.

\subsection{Ivrea Testbed: telemedicine and generic user applications}

The WIND test-bed in Ivrea (Italy) is based on the PMP (Pointto-Multi-Point) WiMAX technology release $\mathrm{d}$ and adopts the Alcatel 7387 equipment (based on Alvarion technology) using two channels $(7 \mathrm{MHz})$ in the $3.5 \mathrm{GHz}$ range: the first channel on $3,4685 \mathrm{GHz}$; the second is $3,4755 \mathrm{GHz}$.

The Ivrea test-bed has been applied to the Telemedicine system scenario of the WEIRD project and other scenarios for generalusage applications, including video communications and digital voice connections (VoIP). All these applications require the availability of a broadband channel and can be hardly enjoyed while in mobility, especially in remote areas. WiMAX technology can solve this issue.

Two different tests have been carried out for the telemedicine applications. 
- Medical information exchange involving ultrasound images test - aimed at evaluating if the transmission of ultrasound pictures from the doctor (at the patient location) to an expert doctor (within the hospital support) has good user experience for make a good diagnostic in a remote visit. The test setup consisted in a portable ultrasound device, notebook mobile client and a FTP server (in the hospital), connected through a WiMAX link. During the test an image has been uploaded on the laptop and sent from the laptop to the server using FTP session.

- Medical video conference test - aimed at evaluating the possibility to hold a videoconference between the patient or the doctor (home) and the doctor in the hospital. The test setup consisted in a PC supporting video call (camera and video call software) and a notebook client that support video calls. The test has been carried out as follow: video call between the PC and the notebook has been established to show the patient situation and discuss medical options.

In particular, the capability of the system to allow the connectivity and the service erogation when the user is moving has been tested. The same tests have been performed in stationary mode and varying the speed of the user in the sector from $0 \mathrm{Km} / \mathrm{h}$ to $90 \mathrm{Km} / \mathrm{h}$ in the urban environment without loosing the transmission for both the transmission channels. As far as concerns FTP and VoIP a various set of test were performed on different conditions (i.e. urban and rural area, LOS and NLOS) considering a wide range of parameters, like the coverage area, radio measurements, throughput, latency and delay. The data-rate has been evaluated taking into account the distance, the radio connection conditions between the BS and the ST, and the capability to adapt the channel selection according to the traffic density. Regarding the coverage of the two sectors, we analysed the capability of the system with the Adaptive Antenna System (AAS) feature on or off. We performed stationary tests: it revealed that the FTP throughput in downlink is quite stable according to the service profile defined for both in uplink and downlink. Regarding the maximum cell capacity in downlink and in uplink, some tests have been done in order to understand the total cell throughput reached respect to the theoretical value for each service profile.

The tests were performed considering the following system parameters: $5 \mathrm{MHz}$ of channel bandwidth, Time Division Duplex (TDD) with the configuration of 2:1 and with AAS on or off, according to the test. The modulations schemes thresholds and the Received Signal Strength Indication (RSSI) are described in Table 3.

Tests were performed with three different types of radio conditions (good, medium and bad) defined as follow: Good radio conditions (RSSI > $-65 \mathrm{dBm})$, Medium radio conditions, -(75 $\mathrm{dBm} \leq \mathrm{RSSI}<-65 \mathrm{dBm})$, Bad radio conditions( RSSI $<-75$ $\mathrm{dBm})$. The following tests have been performed: Network entry/Network entry sensitivity, Coverage tests, Stationary tests including FTP file transfer and ping tests, Capacity tests; Indoor tests. The results of FTP tests are shown in Table 4 (downlink)
Table 3. Modulation schemes and FEC

\begin{tabular}{|l|r|c|}
\hline $\begin{array}{l}\text { Modulation } \\
\text { Scheme and FEC }\end{array}$ & $\begin{array}{l}\text { RSSI minimum } \\
\text { threshold [dBm] }\end{array}$ & $\begin{array}{l}\text { RSSI maximum } \\
\text { threshold [dBm] }\end{array}$ \\
\hline QPSK $1 / 2^{1 / 2}$ & -157.5 & -70 \\
\hline 16QAM $^{3 / 4}$ & -73 & -50 \\
\hline 64QAM $3 / 4$ & -53 & -30 \\
\hline
\end{tabular}

Table 4. Performance of FTP Tests in downlink

\begin{tabular}{|c|c|c|c|c|c|c|}
\hline 莺 & 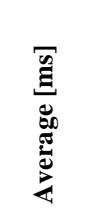 & 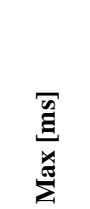 & 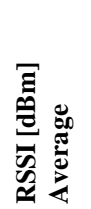 & 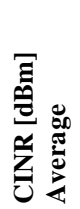 & 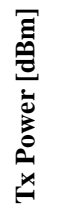 & 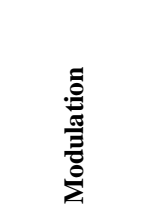 \\
\hline Good & 1467.8 & 1630.5 & -53.10 & 21.69 & 3 & 16 QAM $1 / 2$ \\
\hline Medium & 1302.1 & 1608.2 & -73.88 & 17.27 & 22 & QPSK $1 / 2$ \\
\hline $\mathrm{Bad}$ & 1236.2 & 1619.4 & -77.85 & 11.44 & 23 & QPSK $1 / 2$ \\
\hline
\end{tabular}

Table 5. Performance of FTP Tests in uplink

\begin{tabular}{|c|c|c|c|c|c|c|}
\hline 递 & 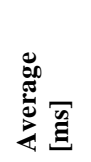 & 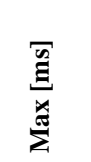 & 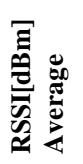 & 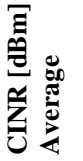 & 离 & 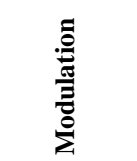 \\
\hline Good & 232.41 & 602.47 & -53.92 & 22.12 & 11.14 & 16 QAM 1/2 \\
\hline Medium & 240.35 & 724.10 & -72.53 & 18.37 & 22.65 & QPSK 1/2 \\
\hline $\mathrm{Bad}$ & 242.07 & 746.43 & -78.51 & 9.94 & 23 & QPSK $1 / 2$ \\
\hline
\end{tabular}

and in Table 5 (uplink). The Average and the Maximum time for the FTP service are reported.

As far as concerns VoIP tests the following evaluations methodologies are used:

- $\quad$ PAMS (Perceptual Analysis Measurement System) - it is based on Human Hearing Factors

- ITU P.861 (Calculating Telephone Quality using Perceptual Speech Quality Measure algorithm)

and both of them are correlated to a Mean Opinion Score MOS score on a $1-5$ scale.

The quality of the VoIP has been tested through the use of a specific equipment to test VoIP quality. The analyzer used was Internet Advisor from Agilent Technologies. This instrument allows running several tests according to the selected methodology:

- PAMS: The Perceptual Analysis Measurement System is based on the algorithms developed originally by British Telecom Labs. PAMS is used in place of traditional audio quality measurements because of the nonlinear nature of voice codecs, and uses auditory and cognitive models. PAMS is designed to provide an objective measure, which predicts the results of subjective listening tests (MOS) on telephony systems.. PAMS provide two different values: Listening Quality 
Table 6. LOS and LES values

\begin{tabular}{|c|c|l|}
\hline Value & Quality of & Listening Effort (LES) \\
\hline 5 & Excellent & Complete relaxation \\
\hline 4 & Good & Attention necessary \\
\hline 3 & Fair & Moderate effort required \\
\hline 2 & Poor & Considerable effort required \\
\hline 1 & Bad & No meaning understood \\
\hline
\end{tabular}

Table 7. Mean Opinion Score

\begin{tabular}{|c|c|}
\hline Value & Quality of \\
\hline 5 & Excellent \\
\hline 4 & Good \\
\hline 3 & Fair \\
\hline 2 & Poor \\
\hline 1 & Bad \\
\hline
\end{tabular}

Table 8. MOS values relacted to PSNR

\begin{tabular}{|c|c|}
\hline Mean Opinion Score & PSNR [dB] \\
\hline 5 (Excellent) & $>37$ \\
\hline 4 (Good) & $31-37$ \\
\hline 3 (Fair) & $25-31$ \\
\hline 2 (Poor) & $20-25$ \\
\hline 1 (Bad) & $<20$ \\
\hline
\end{tabular}

Table 9. MOS, LQS AND LES values

\begin{tabular}{|l|l|l|l|}
\hline Site & MOS & LQS & LES \\
\hline Floor 5 & 3.21 & 3.54 & 3.96 \\
\hline Floor 4 & 3.33 & 3.46 & 3.87 \\
\hline Floor 3 & 3.22 & 3.51 & 3.93 \\
\hline Floor 2 & 3.31 & 3.45 & 3.64 \\
\hline
\end{tabular}

Score (LQS) and Listening Effort Score (LES). The meaning of the values is showed in Table 6 .

- PESQ: Perceptual Evaluation of Speech Quality is based on the ITU-T P.862 standard. This standard defines the algorithms used to compare reference speech samples with test samples to measure quality degradation due to distortion. PESQ is used in place of traditional audio quality measurements because of the nonlinear nature of voice codecs, and uses auditory and cognitive models.

MOS values (see Tables 7 and 8) are obtained using both G711 [7] and G729 [8] codec. Table 9 shows the values of MOS, listening quality score (LQS) and listening effort score (LES) obtained on five different sites. The values show how an acceptable quality can be achieved.

In conclusion, several tests were performed: just to summarise, regarding the coverage of the sector 1 , with the AAS feature on, it reaches 2000 metres in an open area but the coverage in Ivrea historical centre (in the direction of the mail lobe) does not exist due to the streets and buildings configuration. For sector 2, the coverage has reaches 800 metres but one should take into account that there are high buildings in the front of the antenna main lobe.

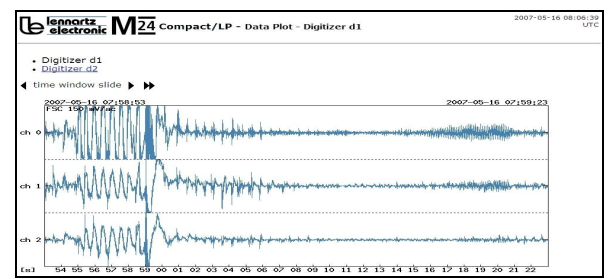

Figure 3. Three-component signal of the seismic station

The stationary tests has revealed that the FTP throughput in downlink is stable when there are good radio conditions but it becomes quite instable when there is a poor received signal strength. Nevertheless, the average throughout is between the expected values, 1.2 and $1.4 \mathrm{Mbps}$, regarding a maximum of $1536 \mathrm{kbps}$ for each user, defined as the service profile of this trial in downlink. In uplink, there are no issues in the throughput, reaching in good or bad radio conditions almost the maximum of $256 \mathrm{kbps}$, defined as the service profile in uplink.

The most surprising WiMax solution tried in this trial was the indoor CPE. It turned to be a stable and useful solution in the indoor coverage since the throughput results in every indoor FTP test were very stable and always at the maximum allowed per user. The same happens in the uplink case and it is very important for the application taken into account.

Regarding the maximum cell capacity, in downlink, it has reached the $88 \%$ of the theoretical value was reached (for 16QAM). In uplink, and taking into account that the service profile was $256 \mathrm{kbps}$, the total cell throughput reached was $75 \%$ of the theoretical value and we think that is a good starting point.

\subsection{Environmental monitoring}

\subsubsection{Volcano Monitoring testbed results}

The test has been realized using a portable digital seismic station Lennartz M24 equipped with a Lennartz LE-3Dlite seismometer. Both the instruments are powered by a $12 \mathrm{~V}$ battery. The data acquisition is on-site on an IDE 2.5" 20 GB hard disk. The Lennartz M24 seismic stations are characterized by both standalone recording and remote data retrieval via serial line or TCP/IP protocol on a web-based interface. Dedicated software allows selecting the data format, the compression type, as well as the time-window length of the seismic signal to download.

During the test, the transmission has been obtained by means of a CPE/antenna WiMAX equipment Alvarion manufactured by Alcatel linked to the LAN output interface of the seismic station. Specific IP address was assigned at the station according to the network properties established for the test-bed.

We have selected a five minutes time-window of the seismic signal to download in compressed ASCII format. The data transfer using the WiMAX technology has been concluded in about six seconds. In this way, it was demonstrated that WiMAX technology can be successfully used for a near real-time remote processing of the data. In a real scenario, this capability allows to integrate the data gathered by the temporary seismic stations with the permanent network ones. 


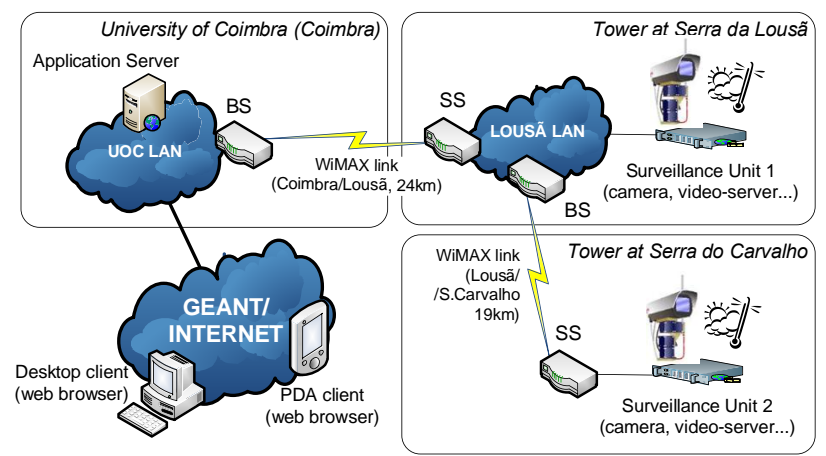

Figure 4. Fire Prevention Test-bed

Fig. 3 shows about 30 seconds of the seismic signal gathered during the test-bed. The oscillations are some jumps performed near the seismograph to simulate an earthquake. As a comparison, it is possible to perform the data download also using the 9600 bps GSM connection via serial output. In this case, the time spent for the transfer of the same seismic signal is about fifteen minutes, which is an unacceptable delay in emergencies cases. Other faster mobile communication systems, such as GPRS or UMTS, do not allow a true advantage because of the weakness of these connections during strong earthquakes or real emergencies. Even the widely-used radio modem links suffer from limited bandwidth, while the Wi-Fi technology cannot be used on long distances.

\subsubsection{Fire Prevention Testbed results}

The fire prevention testbed encompasses two of the fire surveillance towers (Serra da Lousã and Serra do Carvalho, Portugal) already in use by regional fire prevention services. Each of these towers was equipped with surveillance cameras, digital compasses (for geographical location of detected fires), and weather stations (wind, temperature, humidity). Collected data feeds a fire prevention application located in the control center, making it possible to immediately pinpoint potential fires in geographical information databases (allowing better guidance of the helicopter transported first response firefighters) and to save video and data for forensic analysis (for instance for research purposes or criminal investigation).

Fig. 4 presents the topology of the test-bed. A $22 \mathrm{~km}$ WiMAX link connects the University of Coimbra with the first surveillance unit (Serra da Lousã). A second WiMAX link connects Serra da Lousã with Serra do Carvalho (19 km), further stretching WiMAX coverage into the remote forest. Both links are point-to-multipoint (making it possible to add further surveillance units, in the future), and neither of these towers has alternative broadband coverage (ADSL, 3G...).

So far tests focused on functional issues and the validation of the QoS mechanisms of the WEIRD architecture. Currently the system is using the 3443-3471 MHz range for one of the links and the $3543-3571 \mathrm{MHz}$ range for the other. This bandwidth is enough for the traffic generated by the surveillance units (around 1.5 Mbps for surveillance unit), even without aggressive compression mechanisms or pre-processing of the video streams.
Table 10. WiMAX equipments configuration

\begin{tabular}{|c|c|c|c|}
\hline BS or SS, Location & BW $(\mathrm{MHz})$ & Power $(\mathrm{dbm})$ & Antenna \\
\hline BS, UoC & \multirow{4}{*}{$\begin{array}{c}3.5, \\
7 \text { and } 14\end{array}$} & 23 & $90^{\circ}$ \\
\hline SS, SL & & 16 & $15^{\circ}$ \\
\hline $\mathrm{BS}, \mathrm{SL}$ & & 23 & $90^{\circ}$ \\
\hline $\mathrm{SS}, \mathrm{SC}$ & & 16 & $15^{\circ}$ \\
\hline
\end{tabular}

Table 11. Propagation Conditions

\begin{tabular}{|l|r|c|}
\hline \multicolumn{1}{|c|}{ Link direction } & $\begin{array}{c}\text { Distance } \\
(\mathrm{Km})\end{array}$ & Propagation \\
\hline Aggregation_Link & 22.8 & LOS \\
\hline SingleCam_Link & 18.8 & LOS \\
\hline
\end{tabular}

Table 12. Measured Bandwidth for the Aggregation_Link

\begin{tabular}{|c|r|r|r|}
\hline $\begin{array}{l}\text { UoC - SL } \\
\text { Aggregation_Link }\end{array}$ & $\begin{array}{l}\text { Uplink } \\
\text { (Mbps) }\end{array}$ & $\begin{array}{l}\text { Downlink } \\
\text { (Mbps) }\end{array}$ & $\begin{array}{l}\text { Total } \\
\text { (Mbps) }\end{array}$ \\
\hline $3.5 \mathrm{MHz}$ & 1.1 & 1.3 & 2.4 \\
\hline $7 \mathrm{MHz}$ & 2.2 & 2.7 & 4.9 \\
\hline $14 \mathrm{MHz}$ & 4.5 & 5.6 & 10.1 \\
\hline
\end{tabular}

Table 13. Measured Bandwidth for the SingleCam_Link

\begin{tabular}{|c|r|r|r|}
\hline $\begin{array}{l}\text { SL - SC } \\
\text { SingleCam_Link }\end{array}$ & $\begin{array}{l}\text { Uplink } \\
\text { (Mbps) }\end{array}$ & $\begin{array}{l}\text { Downlink } \\
\text { (Mbps) }\end{array}$ & $\begin{array}{l}\text { Total } \\
\text { (Mbps) }\end{array}$ \\
\hline $3.5 \mathrm{MHz}$ & 2.0 & 2.4 & 4.4 \\
\hline $7 \mathrm{MHz}$ & 4.1 & 4.8 & 8.9 \\
\hline $14 \mathrm{MHz}$ & 7.8 & 9.6 & 17.4 \\
\hline
\end{tabular}

Detailed information about the WiMAX equipments configuration is illustrated in Table 10.

In order to study the performance of the installed testbed, a set of measurements has been made for the two WiMAX links - Aggregation_Link between University of Coimbra (UoC) and Serra da Lousã (SL), and the SingleCam_Link between Serra da Lousã (SL) and Serra do Carvalho (SC). The aim of these measurements was to verify if the installed WiMAX links were able to fulfill the fire prevention application bandwidth requirements - cameras will stream video with $1.5 \mathrm{Mbps}$.

It is important to mention the differences between the two radio links. Although both of them have Line of Sight (LoS) propagation conditions, the distance for the Aggregation_Link is larger than the SingleCam_Link (Table 11).

Moreover, since the Aggregation_Link is the "aggregation link" of the testbed, it has to carry the video streaming packets from both surveillance cameras: the camera installed in $S L$ and the one installed in SC. For the SingleCam_Link, since it only has to carry video streamed by the camera installed on $S C$ to $S L$ the bandwidth requirements are lower.

The Iperf tool has been used to load the WiMAX network with packets and measure the maximum available bandwidth. The maximum throughput obtained for the Aggregation_Link is shown in Table 12. 
From Table 12 we can see that the $7 \mathrm{MHz}$ channel bandwidth provides $2.2 \mathrm{Mb} / \mathrm{s}$ of throughput for the uplink channel. In this case, it is possible to carry the video from both cameras, but not with the maximum bit rate $(1.5 \mathrm{Mb} / \mathrm{s}$ per camera). This way, the best solution is to use the $14 \mathrm{MHz}$ channel bandwidth, which provides $4.5 \mathrm{Mb} / \mathrm{s}$ dedicated for the uplink direction, enabling the usage of the video streaming with the maximum video encoding rate. Table 13 presents the throughput obtained for the SingleCam_Link.

Since the SingleCam_Link distance is lower than the Aggregation_Link, the measured bandwidth is higher. Only the video from one camera is streamed towards this link. Therefore, the 3.5 $\mathrm{MHz}$ channel bandwidth is sufficient to satisfy the Fire Prevention application bandwidth requirements $(1.5 \mathrm{Mb} / \mathrm{s})$.

\section{CONCLUSIONS}

In this paper we described the main characteristics of the testbeds used in WEIRD project, the test carried on and the results of such test-beds. The test-beds permit to validate all the WEIRD project solutions including architectural (e.g., software modules, signalling, interfaces, ...), and procedural ones (e.g., resources management over the WiMAX link). In particular the results obtained demonstrate the viability of WEIRD solutions on the WiMAX technology to assure QoS for a variety of applications, in details for: environmental monitoring, telemedicine and generic user applications (VoIP and FTP). Future work will comprise the execution of further tests to verify the interoperability of the proposed solutions with other networks, considering end-to-end issues like end-to-end QoS with DiffServ network.

\section{ACKNOWLEDGMENTS}

The authors would like to thank members of WEIRD team who supported this work by participating to WEIRD test-bed setup.

\section{WEIRD DISCLAIMER}

The work described in this paper is based on result of IST FP6 Integrated Project WEIRD. WEIRD receives research funding from the European Community's Sixth Framework Programme. Apart from this, the European Commission has no responsibility for the content of this paper. The information in this document is provided as is and no guarantee or warranty is given that the information is fit for any particular purpose. The user thereof uses the information at its sole risk and liability.

\section{REFERENCES}

[1] Castellano M., Buonocunto C., Capello M., and M. La Rocca, "Seismic surveillance of active volcanoes: the Osservatorio Vesuviano Seismic Network (OVSN - Southern Italy)", Seism. Res. Lett., 73, 2, pp. 177-184, 2002.

[2] G. Ash, et al., "QoS NSLP QSPEC Template”, Internet Draft, December 2006
[3] GEANT2 web site, http://www.geant2.net/

[4] IEEE Standard for Local and Metropolitan Area Networks. Part 16: Air Interface for Fixed Broadband Wireless Access Systems, IEEE STD 802.16 2004, October 2004.

[5] IEEE Standard for Local and Metropolitan Area Networks. Part 16: Air Interface for Fixed Broadband Wireless Access Systems. Amendment 2: Physical and Medium Access Control Layer for Combined Fixed and Mobile Operation in Licensed Bands, IEEE P802.16e/D12, February 2005.

[6] IETF 16 ng Working Group

http://www.ietf.org/html.charters/16ng-charter.html

[7] ITU-T Reccommendation G.711 (extract from the blue book) General Aspects of Digital Transmission Systems: Terminal Equipments - Pulse Code Modulation (PCM) of voice frequencies

[8] ITU-T, G.729 : Coding of speech at $8 \mathrm{kbit} / \mathrm{s}$ using conjugatestructure algebraic-code-excited linear prediction (CSACELP)

[9] ITU-T G.1010 - End-user multimedia QoS categories.

[10] G. Saccorotti, S. Petrosino, F. Bianco, M. Castellano, D. Galluzzo, M. La Rocca, E. Del Pezzo, L. Zaccarelli, and P. Cusano, "Seismicity associated with the 2004-2006 renewed ground uplift at Campi Flegrei caldera, Italy". Phys. Earth Planet. Int., 2007, doi:10.1016/j.pepi.2007.07.006

[11] J. Case, M. Fedor, M. Schoffstall, J. Davin, "A Simple Network Management Protocol (SNMP)”, RFC 1157, May 1990.

[12] J. Manner, G. Karagiannis, A. McDonald, "NSLP for Quality-of-Service Signalling”, Internet Draft, October 2006

[13] J. Rosenberg, et al., "SIP: Session Initiation Protocol", RFC 3261, June 2002

[14] M. Handley, et al., "SDP: Session Description Protocol", RFC 4566, July 2006

[15] P. Calhoun, et al., "Diameter Base Protocol”, RFC 3588, September 2003.

[16] R. Scarpa and R.I. Tilling (Eds.), "Monitoring and mitigation of volcanic hazards". Springer-Verlag Berlin Heidelberg, 1996, pp. 845.

[17] R. Hancock, et al., (2005). RFC 4080 - Next Steps in Signalling (NSIS): Framework, Internet Engineering Task Force.

[18] Tuomas Nissila, Jyrki Huusko, Ilkka Harjula, Marcos Katz, "Adapter Implementation Between WiMAX Specific Layers and Network/Application Layers", $1{ }^{\text {st }}$ Broadband Wireless Access Workshop, Cardiff, Wales, September 2007.

[19] WEIRD Project web site, http://www.ist-weird.eu/

[20] WiMAX Forum Web site, http://www.wimaxforum.org 